\title{
Pesquisa epidemiológica da saúde do trabalhador Uma reflexão teórica
}

\section{Epidemiologic research in health worker: a reflection theory}

\author{
Julia Trevisan Martins ${ }^{1}$; Renata Perfeito Ribeiro ${ }^{2}$; Maria Cristina Cescatto \\ Bobroff3; Maria Helena Palucci Marziale; ${ }^{4}$ Maria Lucia do Carmo da Cruz \\ Robazzi ${ }^{5}$
}

\section{Resumo}

Uma das formas de se intervir nos riscos que provocam agravos e doenças nos trabalhadores é por meio de pesquisas que investiguem as reais causas e soluções desses problemas. Este estudo de natureza reflexiva tem como objetivo demonstrar os métodos de pesquisas utilizados em epidemiologia. A aplicação de métodos epidemiológicos é fundamental para as diversas áreas da saúde e imprescindível para a investigação relacionada à saúde dos trabalhadores, quer seja em saúde pública, na gestão em saúde ou na prática clinica. Os principais métodos para essa investigação são as pesquisas ou estudos epidemiológicos, os estudos descritivos e analíticos, experimental, coorte, caso-controle, transversal, ecológico, caso, grupos, survey, bibliográfico e documental.

Palavras-chave: trabalhadores; saúde do trabalhador; métodos epidemiológicos; epidemiologia.

\begin{abstract}
One of the ways to intervene in the risks that cause injuries and illnesses among workers is through research that uncovers the real causes and solutions to these problems. This reflexive nature study aims to demonstrate the research methods used in epidemiology. The application of epidemiological methods is fundamental to all areas of health and essential for research related to health of workers, whether in public health, health management or clinical practice. The main methods for this research are epidemiological studies, descriptive and analytical studies, experimental, cohort, case-control, crosssectional, ecological, case groups, and survey, bibliographic and documentary.
\end{abstract}

Keywords: workers, worker health, epidemiological methods, epidemiology.

\footnotetext{
${ }^{1}$ Doutora em Enfermagem Fundamental. Professor adjunto da Universidade Estadual de Londrina - UEL. Email: jtmartins@uel.br

${ }^{2}$ Doutora em Enfermagem. Professor adjunto da Universidade Estadual de Londrina - UEL. Email: perfeitorenata@gmail.com

${ }^{3}$ Doutora em medicina e Ciências da Saúde. Professor adjunto da Universidade Estadual de Londrina - UEL. Email: crisbob@uel.br

${ }^{4}$ Doutora em Enfermagem. Professor titular da Escola de Enfermagem de Ribeirão Preto da Universidade de São paulo - USP.

${ }^{5}$ Doutora em Enfermagem. Coordenadora do programa de pós-graduação mestrado profissional em tecnologia e inovação em Enfermagem da Escola de Enfermagem de Ribeirão Preto da Universidade de São Paulo - USP.
} 


\section{Introdução}

A epidemiologia é um eixo da área de Saúde Pública (SP) que consiste no estudo de doenças transmissíveis e nãotransmissíveis, do conhecimento sobre a morbidade e mortalidade, da frequência dos agravos, entre outros. Esse conhecimento permite um elo entre a comunidade e o governo para a prática da cidadania. Dessa maneira exerce-se o controle da realidade por meio de resultados de pesquisas cujas fontes provêm do próprio ambiente e das pessoas envolvidas. Estabelecem-se, assim, adequações nas políticas públicas de prevenção e controle dos agravos (ROUQUAYROL; GOLDBAUM, 2003).

Segundo Briz (2009) a epidemiologia produz o conhecimento estudando as manifestações da saúde e da doença bem como seus determinantes enquanto a SP beneficia-se dessa, organizandose e planejando ações voltadas para a prevenção de doenças e agravos, promovendo a saúde da população. No entanto, atualmente, há uma interface entre a epidemiologia e a SP considerando-se que a primeira também tem aplicado o conhecimento das pesquisas para o controle, promoção e prevenção da saúde.

Enfatiza-se que, como ciência, a epidemiologia busca, através da explicação de determinados fatos e eventos, estudar o processo saúde-doença analisando a distribuição das enfermidades, danos e agravos à saúde em coletividades humanas. Como método, a epidemiologia tem o propósito de fornecer subsídios permanentes para a tomada de decisões que visem beneficiar a saúde da população (SILVA, 2008).

Buscando informações, a epidemiologia relaciona a saúde ao processo de trabalho e, nesse contexto, revela, descreve e analisa características comuns e incomuns das repercussões sanitárias em diferentes grupos de trabalhadores. Assim, resgata a análise da "heterogeneidade dos grupos homogêneos" de trabalhadores (SOUZA, 2012).

Neste estudo, entende-se como trabalhadores aqueles que exercem atividades para sustento próprio e/ou de seus dependentes, seja no mercado de trabalho formal ou informal; os que trabalham como assalariados, domésticos, rurais, autônomos, temporários, servidores públicos, cooperativados, empregadores; aquele não remunerado que trabalha no domicílio, o aprendiz ou estagiário; e aqueles que estão afastados temporariamente ou definitivamente do mercado de trabalho por doença, aposentadoria ou desemprego (BRASIL, 2004).

Com a promulgação da Lei Orgânica de Saúde em 1990, a saúde do trabalhador (ST) foi incorporada às ações do Sistema Único de Saúde (SUS), e desta maneira, conferida ao SUS a responsabilidade de coordenar as políticas desta área, tendo como componentes estruturais a Vigilância Epidemiológica e Sanitária (BRASIL, 2011).

A incorporação do campo da ST ao SUS agregou-se à Vigilância em Saúde, para ampliar o olhar sobre as relações saúde-trabalho nas práticas da Vigilância Sanitária. Reforçou-se, dessa maneira, a fiscalização dos processos e dos ambientes de trabalho, conferindo ao SUS uma nova visão sobre as fontes de adoecimento da população no seu próprio local de sua produção (VASCONCELLOS et al, 2009).

Diante dessa nova concepção, a área de ST passa a ser estruturada no âmbito do SUS, e surge um campo de rediscussão de seu conceito e abrangência, principalmente no que se refere à investigação dos determinantes dos agravos à saúde, comumente mensurados através da frequência dos acidentes e das doenças ocupacionais. Decorre daí uma reformulação na estratégia de Vigilância dos Agravos que tem gênese na relação produção/saúde (VASCONCELOS; RIBEIRO, 1997).

Assim, por força da lei e de um novo entendimento de ST, que foi pauta de ampla discussão no movimento da reforma Sanitária Brasileira, o SUS passa então a ter uma atribuição holística para a área de saúde e para a ST, entendendo a assistência, a pesquisa, a sistematização das informações e as 
intervenções sobre os determinantes do risco, como construtores de um novo paradigma de saúde.

Desta forma, surgem as diretrizes das ações de Vigilância em Saúde do Trabalho (VST) que pressupõem a universalidade e integralidade das ações, entendendo a assistência e recuperação dos agravos, e a prevenção por meio de intervenções nos processos de trabalho; a plurinstitucionalidade, por meio de ações articuladas entre as instâncias de vigilância em ST, centros de atendimento e assistência, instituições, universidades e centros de pesquisa sobre saúde e ambiente; o controle social, com a participação dos trabalhadores e de seus representantes, em todas as etapas da vigilância em ST; a hierarquização e descentralização; a interdisciplinaridade, abrangendo as diversas áreas de conhecimento técnico com o saber operário; a pesquisa e intervenção (processo no qual a pesquisa é parte integrante e indissolúvel que subsidia e aprimora a própria intervenção); e, o caráter transformador dos determinantes e condicionantes dos problemas de saúde relacionados aos processos e ambientes de trabalho (BRASIL, 1998).

Denota-se que a VST vem se firmando em diversas áreas de conhecimento e principalmente na SP e nesse sentido, tem se preocupado com as mudanças de entendimento do processo saúde/ doença, com a finalidade de dar respostas efetivas aos problemas que afetam os trabalhadores que são complexos e de difícil resolução mediante ações somente curativas e preventivas (ALVES, 2003)

Ressalta-se ainda a Vigilância Ambiental considerada um novo campo do conhecimento, que trata da compreensão e da análise dos condicionantes ambientais que afetam a saúde humana. É uma área da SP que envolve a pluricausalidade, na interação de múltiplos fatores que interagem e são interdependentes e que podem determinar a ocorrência de agravos e/ou doenças nas populações expostas (AUGUSTO, 2003).

Enfatiza-se que as abordagens cartesianas e positivistas derelaçõesmonocausais entre exposição- efeito devem ser substituídas ou repensadas, daí a importância da Vigilância Ambiental em ST. Neste contexto, e no âmbito do SUS, a promoção da saúde deve considerar o ambiente no qual as pessoas vivem e trabalham (AUGUSTO, 2003).

Sabe-se que uma das formas de intervenção nos fatores que provocam o agravo e a doença do trabalhador é por meio de pesquisas que investiguem as reais causas e soluções desses problemas. Assim, este estudo reflexivo tem como objetivo demonstrar os métodos de pesquisas utilizadas em Epidemiologia na área de ST, visto que é de importância ímpar, nas mais diversas áreas da saúde, e também fundamental no dia a dia dos profissionais da saúde e de áreas afins, quer seja no ambiente da SP, da gestão em saúde, ou da prática clinica, dentre outros.

\section{Pesquisa ou Estudos Epidemiológicos}

O método ou desenho epidemiológico é um instrumento de produção de conhecimento que, na área de ST contribui substancialmente para a promoção da saúde e prevenção de agravos dessa população específica. Estudos epidemiológicos avaliam a exposição a um determinado risco e os efeitos dessa exposição à saúde das pessoas, particularmente de grupos específicos ou expostos aos mesmos riscos e/ou agravos. Estes estudos são a base para o conhecimento da realidade de saúde de uma população e para o planejamento de ações preventivas.

Há divergências sobre os desenhos (delineamentos ou designs) desses estudos, assim, para facilitar a compreensão do leitor, os mesmos serão apresentados em estudos descritivos e estudos analíticos. Estudos epidemiológicos descritivos buscam identificar um padrão entre os casos estudados e abrangem o tempo, o lugar e o indivíduo. Assim, epidemiologistas utilizam os estudos descritivos para formular hipóteses, mas raramente testam-nas (U.S. DEPARTMENT OF HEALTH AND HUMAN SERVICES, 2012). 
Para testar as hipóteses, utilizam os estudos analíticos epidemiológicos que buscam as causas, os efeitos e como a doença ou o agravo ocorreram comparando os grupos. Desta maneira, quantificam a associação entre a exposição e o desfecho testando hipóteses causais. A epidemiologia por si própria não prova se uma exposição particular causou um desfecho particular, mas apresenta evidências importantes para o controle e para tomada de medidas preventivas. Assim, os estudos epidemiológicos abrangem duas categorias, os experimentais e os observacionais (U.S. DEPARTMENT OF HEALTH AND HUMAN SERVICES, 2012).

Nesse contexto, para melhor compreensão serão destacadas algumas diferenças básicas entre estudos descritivos e estudos analíticos na área da saúde.

\section{Estudos descritivos}

Têm como finalidade informar sobre a distribuição de um evento, na população, em termos quantitativos podendo ser de incidência (casos novos) ou de prevalência (casos existentes). Há cinco questões fundamentais que se buscam responder em um estudo descritivo, que em inglês são denominados os $5 \mathrm{~W}$ (what, who, where, when, why/how), a saber: o que (problema de saúde investigado), quem (indivíduo), onde (local) quando (tempo) e porque/como (causas, fatores de risco, modos de transmissão) (U.S. DEPARTMENT OF HEALTH AND HUMAN SERVICES 2012).

O principal objetivo da pesquisa descritiva é o de descrever características de determinada população ou fenômeno ou estabelecer a relação entre as variáveis. Uma das características mais significativas desse tipo de estudo é a utilização de técnicas padronizadas de coleta de dados tal como o questionário (GIL, 2008). Esse tipo de estudo tem dois objetivos, o primeiro é identificar grupos de risco, isto é, informar sobre as necessidades e as características dos segmentos que poderiam beneficiar-se, de alguma maneira, com medidas preventivas. Portanto, tem uma relação direta com a epidemiologia no que diz respeito à prevenção de doenças e planejamento de saúde. O segundo objetivo é buscar explicações para as variações de frequência dos agravos, o que serve de base para o prosseguimento de pesquisas sobre o assunto, por meio de estudos analíticos (PEREIRA, 2001).

$\mathrm{Na}$ pesquisa descritiva, apesar de estudar as relações entre duas ou mais variáveis, o pesquisador observa os fatos, registra-os, analisaos e correlaciona-os aos fenômenos ou às outras variáveis, mas não interfere neles (os fenômenos são estudados, mas não são manipulados pelo pesquisador) (GIL, 2008; CERVO et al, 2007). Dessa maneira, nos estudos descritivos não existe a formação de grupo-controle para comparar os resultados e, devido a essa peculiaridade, são considerados estudos não controlados (PEREIRA 2001).

Esses estudos buscam responder o tempo, o lugar e/ou as características dos sujeitos da amostra (idade, sexo, escolaridade, renda, ocupação, fatores de risco, doenças crônicas associadas, etc) para compreender quando, onde e quem adoece numa dada população (LIMA-COSTA; BARRETO, 2003).

Em estudos descritivos normalmente resgatamse dados pré-existentes, também denominados de estudos retrospectivos. Esses dados podem ser resgatados de fontes primárias, secundárias ou terciárias (LIMA-COSTA; BARRETO, 2003). Entende-se por fontes primárias: documentos primários, considerados aqueles originais e publicados pela primeira vez pelos autores. Do ponto de vista de procedência - aqueles que contêm a informação original - como, por exemplo, monografias, dissertações, teses, livros, relatórios técnicos e científicos, artigos publicados em revistas científicas, anais de congressos, informações governamentais, acadêmicas, de negócios e da 
indústria em formato impresso ou eletrônico (PELLIZON et al, 2003). Por outro lado, fontes secundárias contêm trabalhos não originais e que basicamente citam, revisam e interpretam trabalhos originais. Incluem-se nessa categoria a revisão bibliográfica e a revisão sistemática da literatura, os livros-texto, tratados, enciclopédias, artigos de divulgação, índices, bases de dados referenciais, diretórios, entidades e eventos da área da saúde, além dos serviços de informação relacionados a essas fontes (PELLIZON et al, 2003). As fontes terciárias contêm índices categorizados de trabalhos primários e secundários, com ou sem resumo, citamse como exemplos as bases de dados bibliográficos, índices e listas bibliográficas (PELLIZON et al, 2003).

Tanto para os estudos descritivos como para os analíticos há que se fazer uma busca dessas fontes de dados para discutir os vários aspectos dos resultados dessas pesquisas no âmbito da literatura científica. Para isso, na área da saúde, utiliza-se um componente integrador via internet, registrados como Descritores em Ciências da Saúde (DeCS) criados pela Biblioteca Virtual em Saúde (BIREME). Os termos autorizados em Ciências da Saúde são registrados nessa base de dados e são também denominados como palavras-chave, uni termos ou descritores. Essas palavras-chaves são estruturadas em três idiomas - português, inglês, espanhol para a indexação de artigos científicos e constituise em uma maneira de integração entre as bases de dados da internet sendo que a busca por termos ou palavras não autorizadas pode inviabilizar o resgate dos artigos sobre o tema da pesquisa em questão, comprometendo a discussão sobre o assunto tratado (PELLIZON et al, 2003). Tal terminologia utilizou como base o Medical Subject Headings (MeSH) da Biblioteca Nacional de Medicina dos Estados Unidos objetivando uma terminologia "comum para pesquisa em três idiomas, proporcionando um meio consistente e único para a recuperação da informação independente do idioma (PELLIZON et al, 2003, p.495).

\section{Estudos analíticos}

São estudos que buscam esclarecer uma dada associação entre uma exposição, em particular, e um efeito específico (doença ou condição relacionada à saúde). Esses estão normalmente subordinados a uma ou mais questões científicas, às hipóteses, que relacionam eventos: uma suposta causa e um dado efeito, ou exposição e doença, respectivamente. Por exemplo: exposição a um dado é causa de obesidade, onde a obesidade é a doença, tendo como efeito o diabetes.

Uma consideração importante acerca dos desenhos de pesquisa observacionais e dos desenhos experimentais é a "posição do investigador perante o seu objeto de estudo" que define o tipo de desenho a ser realizado (ALMEIDA FILHO; ROUQUAYROL, 2006).

Enfatiza-se que os principais delineamentos de estudos analíticos são o estudo ecológico, transversal ou seccional, o de caso-controle (casoreferência) e o estudo de coorte prospectivo (LIMACOSTA, BARRETO, 2003)

\section{Abordagens Epidemiológicas em Saúde do Trabalhador}

Apresentam-se na sequência uma rápida explanação das principais abordagens Epidemiológicas comumente utilizadas em ST no Brasil.

\section{Estudo Experimental (Ensaio Clínico Randomizado, Estudo de Intervenção)}

Neste tipo de estudo o pesquisador é o responsável pela exposição dos indivíduos no próprio ambiente em que vivem e trabalham, utilizando uma medida preventiva que modifique os efeitos, aos indivíduos, frente às exposições (Ex.: vacina, processo educativo, mudança de hábitos) ou a exposição de animais aos fatores de risco em 
laboratório (LIMA-COSTA, BARRETO, 2003).

É, portanto, um estudo longitudinal porque tem seguimento, ou seja, os indivíduos (sujeitos da amostra) são seguidos por um determinado tempo para a verificação/constatação dos efeitos.

Nessa perspectiva, o experimento tem como objetivo a realização de teste de variáveis ou condições impostas a grupo chamado de experimental, comparando-o a outro grupo que é mantido em suas condições habituais e é chamado de grupo controle sendo que ambos os grupos que farão parte do estudo são obtidos de amostras aleatórias (FLETCHER et al, 1996).

$\mathrm{Na}$ área da saúde, o modelo clássico de estudo experimental é o ensaio clínico randomizado sendo, inclusive considerado o melhor delineamento de pesquisa (FLETCHER et al, 1996). A randomização é uma maneira de tornar os grupos (o grupo experimental e o de controle) comparáveis e evitar vieses. Outra característica que amplia o impacto metodológico desse delineamento é a possibilidade de mascaramento ou cegamento dos sujeitos do estudo, isto é, o fato de se conhecer que um sujeito é do grupo controle pode alterar as reações dos mesmos e, também, a dos pesquisadores. Quando apenas os sujeitos da pesquisa desconhecem o grupo ao qual pertencem, o estudo é conhecido como cego simples ou unicego; quando os pesquisadores e os sujeitos desconhecem o experimento, é conhecido como duplo-cego (CAMPBELL; STANLEY, 1966; ALMEIDA FILHO; ROUQUAYROL, 2006). Denota-se que nem sempre é possível realizar o cegamento, devido ao tipo de intervenção ou por motivos éticos como, por exemplo, comparações entre técnicas de cirurgia ou uso de medicamentos. Quando não for possível o cegamento o estudo é conhecido como sendo aberto (ALMEIDA FILHO; ROUQUAYROL, 2006).

É importante destacar algumas limitações de estudos experimentais que, em alguns casos, implicam na exposição de seres humanos a certas intervenções ou fatores de risco à sua saúde, implicando em restrições éticas que inviabilizam tal desenho. Por outro lado há também fatores positivos na introdução ou remoção de certos fatores em um determinado grupo de indivíduos e quando essas intervenções provocam a melhoria da saúde ou a diminuição da incidência de doenças consideram-se ensaios quase experimentais (ALMEIDA FILHO; ROUQUAYROL, 2006).

Ressalta-se que as pesquisas quase experimentais são desenvolvidas com idêntico rigor metodológico ao das pesquisas experimentais. Nesses casos, a comparação entre as condições estudadas pode ser realizada com grupos não equivalentes ou com os mesmos sujeitos antes de uma determinada intervenção. Uma limitação das pesquisas quase experimentais é a perda da capacidade do investigador em controlar rigorosamente "o que ocorre a quem". Por outro lado, consegue-se "observar o que ocorre, quando ocorre, a quem ocorre, tornando-se possível, de alguma forma, a análise de relações causa-efeito" (GIL, 2008). Como exemplo, em ST, podem-se comparar os índices de absenteísmo dos trabalhadores de uma determinada empresa antes e após a implantação de melhorias para os próprios profissionais. Nesse caso "não houve um experimento propriamente dito, mas um quase experimento" (GIL, 2008).

\section{Estudo de Coorte}

É um tipo de estudo analítico observacional longitudinal que visa acompanhar dois grupos de indivíduos e compará-los, verificando se os expostos a um determinado fator de risco desenvolvem uma doença em maior ou menor proporção que aqueles não expostos ao fator de risco (SOARES; SIQUEIRA, 1999; PEREIRA, 2001).

É uma investigação que segue o curso natural dos acontecimentos partindo da exposição em direção ao efeito da mesma; considera-se, assim, a cronologia dos acontecimentos (PEREIRA, 2001). Neste estudo acompanha-se esse grupo de 
pessoas por um determinado tempo e procura-se identificar a incidência de uma determinada doença ou ocorrência relacionando-a ao fator de risco de exposição (SOARES; SIQUEIRA, 1999).

Podem ser de dois tipos: prospectivo ou estudo de coorte concorrente e retrospectivo ou estudo de coorte histórica. São também chamados de estudo de seguimento ou follow-up porque o investigador segue os sujeitos da amostra por um determinado tempo (ALMEIDA FILHO; ROUQUAYROL, 2006). No estudo de coorte prospectivo a "coorte é acompanhada desde o momento da exposição" sendo que o investigador procede aos registros das ocorrências conforme as mesmas vão acontecendo até a data prevista de encerramento da investigação (ALMEIDA FILHO; ROUQUAYROL, 2006).

Os estudos de coorte são considerados como o melhor tipo de delineamento de pesquisa epidemiológica, pois há possibilidade de calcular estimativas dos índices de incidência (DOCHERT et al, 1993). Uma das características fundamentais desse tipo de estudo é a determinação do tempo de acompanhamento do grupo estudado. Os sujeitos da pesquisa somente entrarão no período de acompanhamento quando estiverem expostos ao fator causal, sendo imprescindível considerar o período latente entre a exposição e a possível ocorrência do evento esperado. Porém, quando esses períodos são desconhecidos, o acompanhamento deve iniciar-se logo após a exposição (ALMEIDA FILHO; ROUQUAYROL 2006).

\section{Estudo de Caso-controle}

É um tipo de estudo analítico observacional longitudinal retrospectivo que parte do efeito para a(s) causa(s) do mesmo. Dessa maneira, os sujeitos são escolhidos porque apresentam a doença em estudo (denominados casos) e são comparados com outros que não possuem essa mesma doença (denominados controles). Investiga-se se ambos os grupos foram expostos aos mesmos fatores de risco para determinar se tais fatores contribuíram para a ocorrência da doença investigada (PEREIRA, 2001). É concebido "especialmente para investigar associações etiológicas em doenças de baixa incidência e/ou condições com período de latência prolongado" (ALMEIDAFILHO; ROUQUAYROL, 2006, p.193).

É também denominada como uma pesquisa $e x-$ post facto porque a exposição ao fator de risco e a doença já ocorreram. Seu propósito é o mesmo que o da pesquisa experimental, ou seja, verificar a existência de relação entre as variáveis e o seu planejamento também é semelhante. Na pesquisa ex-post facto o pesquisador não tem controle sobre a variável independente porque o fenômeno já ocorreu (GIL, 2008).

Esse estudo inicia-se pelo desfecho ou efeito e se busca o fator ou a causa. Não fornecem medidas de ocorrência, prevalência ou incidência do evento a ser investigado, visto que têm uma característica anteriormente conhecida e estabelecida, ou seja, não se obtêm com o processo de investigação (DOCHERT, 1993).

O estudo de caso-controle limita o número de sujeitos participantes da pesquisa, pois se buscam sujeitos controles para os casos de estudo. Assim, este delineamento fragmenta a realidade da população a ser investigada (DOCHERT, 1993).

\section{Estudo Transversal (Seccional, Cross Sectional, Estudo de Prevalência)}

É um tipo de estudo analítico observacional que produz um retrato instantâneo da situação, num determinado momento. A variável de desfecho e a preditiva são observadas no mesmo momento. Utilizado quando a exposição é relativamente constante no tempo e a doença é crônica. Apresenta outras denominações: "seccional, corte, cortetransversal, vertical, pontual ou prevalência" (PEREIRA, 2001). 
Nessa modalidade de investigação a causa e o efeito são identificados simultaneamente, ou seja, é a análise dos dados que possibilita identificar os grupos de interesse, expostos, não expostos, doentes e sadios, de modo a investigar a associação entre exposição e doença. A associação é feita como no risco relativo, entretanto denomina-se razão de prevalência (relação entre a prevalência de expostos e não expostos) e pode ser feito o cálculo também pelo odds ratio (PEREIRA, 2001).

\section{Estudo Ecológico}

Trata-se de um estudo observacional descritivo que tem como delimitador uma área geográfica ou um grupo populacional e, por isso, também é comum ser chamado de estudo populacional. Esse tipo de estudo tem como objetivo comparar indicadores, sendo que a população pode ter tamanho variado, visto que, os dados utilizados são sempre índices relativos e não dados absolutos (GREENLAND; ROBINS, 1994).

Estes estudos comparam a ocorrência da doença/ condição relacionada à saúde e a exposição de interesse entre agregados de pessoas, como por exemplo: populações de determinados países, regiões e municípios, com a finalidade de verificar a possível existência de associação entre elas.

Nos estudos ecológicos típicos, medidas de agregados da exposição à doença são comparadas. Enfatiza-se que não há informações sobre a doença e a exposição do indivíduo e sim do grupo (população) como um todo. Nesse aspecto, são também denominados estudo de grupos, de agregados, de conglomerados, estatísticos ou comunitários ( PEREIRA, 2001). Assim, os indicadores de cada "área ou bloco constituem-se em médias referentes à sua população total, tomada como um agregado integral" (ALMEIDA FILHO; ROUQUAYROL, 2006, p.172).

Para a área de saúde o estudo ecológico serve de base para os estudos das condições de saúde local, regional ou nacional, pois permite comparações como taxas de fecundidade, morbidade, mortalidade, dentre outras. Ressalta-se, porém, que tais estudos, são importantes basicamente, por serem geradores de novas hipóteses (GREENLAND; ROBINS, 1994).

\section{Estudo de Caso}

É um delineamento de pesquisa qualitativa muito utilizado em ciências sociais, "estudo amplo e exaustivo de um ou de poucos objetos, de maneira a permitir seu amplo e detalhado conhecimento", e "a rigor, originou-se no campo da medicina e tem sido intensamente utilizado no estudo de novas doenças e na investigação das causas de doenças raras" (GIL et al, 2006).

O estudo de caso pode ser utilizado para o ensino ou para a pesquisa. Os casos são utilizados como técnicas didáticas para alcançar os objetivos educacionais. Na pesquisa auxiliam no estudo de uma situação problemática e buscam testar uma determinada hipótese formulada pelo pesquisador. Uma das etapas deste tipo de estudo é a definição ou delimitação da unidade-caso. Para se definir a unidade caso, traçam-se os limites do objeto, sendo que isso implica em uma construção intelectual (GIL, 2006).

Os estudos de caso são estudos observacionais exploratórios que são úteis para documentar situações especificas e/ou particulares. Podem ser utilizados como uma primeira abordagem de um tema com a finalidade de avaliar problemas ainda mal conhecidos nos quais as características ou variações naturais não foram detalhados com profundidade. Ressalta-se que uma das formas mais comuns de se construir um estudo de caso é por meio da obtenção da história de vida (PEREIRA, 2008). 


\section{Estudo de Grupos}

São estudos observacionais exploratórios não controlados que visam buscar características peculiares e universais de grupos de pessoas, com uma ou mais características comuns. Assim, este delineamento tem a característica de considerar o grupo como unidade do estudo e não o individuo em particular (ALMEIDA FILHO; ROUQUAYROL,2006). Enfatiza-se que, metodologicamente, não há um número ideal de indivíduos para compor um grupo; a quantidade depende de: tipos de variáveis, propósito do estudo, magnitude dos fenômenos observados e tipo de avaliações utilizadas. Este tipo de estudo é de fácil aplicabilidade e baixo custo. Porém permite somente levantar hipóteses, e, assim, as conclusões são raras.

\section{Levantamento ou Survey}

Nesse delineamento interrogam-se diretamente os sujeitos (grupo de pessoas) cujo comportamento (problema estudado) se deseja conhecer. São úteis para o conhecimento de opiniões, crenças e atitudes. Após a coleta de dados procede-se a análise quantitativa para saber as conclusões acerca dos mesmos (GIL, 2008).

Considera-se que, quando se coletam informações "de todos os integrantes do universo pesquisado, tem-se um censo. Pelas dificuldades materiais que envolvem sua realização, os censos só podem ser desenvolvidos pelos governos ou por instituições de amplos recursos" (GIL, 2008, p.55).

As principais vantagens dos levantamentos são: conhecimento direto da realidade, pois a investigação é "[...] mais livre de interpretações calcadas no subjetivismo dos pesquisadores"; econômica, rápida e com custos relativamente baixos; os dados obtidos podem ser analisados estatisticamente, e podem-se fazer correlações entre as variáveis e até a margem de erro dos resultados (quando as amostras são probabilísticas) (GIL, 2008).
É uma importante limitação a ênfase na percepção que as pessoas têm acerca de si mesmas, pois há "muita diferença entre o que as pessoas fazem ou sentem e o que elas dizem a esse respeito"; "limitada apreensão do processo de mudança" porque, no geral, este delineamento, proporciona uma visão estática do fenômeno estudado ("fotografia de determinado problema, mas não indica suas tendências à variação e muito menos as possíveis mudanças estruturais") (GIL, 2008). Assim, para superar tais limitações, há os levantamentos do tipo painel cuja característica é a coleta de dados de uma determinada amostra ao longo do tempo, com o seguimento dos sujeitos. Há, porém, limitações por mudança de endereço, por resistência e/ou fadiga ao responder aos questionamentos, entre outros aspectos (GIL, 2008).

\section{Estudo Bibliográfico e Pesquisa Documental}

Nesse tipo de estudo utilizam-se dados bibliográficos já publicados para realizar revisões, sínteses ou metanálises. Sendo que, nos estudos de revisão busca-se compilar a produção existente sobre um determinado tema. Nos trabalhos de síntese a finalidade é encontrar os pontos de convergência entre as produções científicas independentes, com o objetivo de produzir novos conhecimentos a partir dos já desenvolvidos. Na metanálise reavaliam-se dados já publicados com a finalidade de aumentar o volume de indivíduos submetidos a uma dada intervenção, em inúmeros trabalhos não relacionados entre si, no que se refere à execução (ALMEIDA FILHO; ROUQUAYROL,2006)

$\mathrm{Na}$ pesquisa documental seguem-se as mesmas etapas da pesquisa bibliográfica. $\mathrm{O}$ primeiro passo é a exploração dos "documentos de primeira mão", aqueles que não receberam qualquer tratamento analítico (documentos oficiais, reportagens de jornal, cartas, contratos, diários, filmes, fotografias, gravações etc.) e os "documentos de segunda 
mão, que de alguma forma já foram analisados" (relatórios de pesquisa, relatórios de empresas, tabelas estatísticas) (GIL, 2008).

\section{Conclusão}

Foram apresentados, neste ensaio, os estudos analíticos utilizados em pesquisas epidemiológicas que são destinados a investigar as relações causais entre eventos, como exposição e doença, ou intervenção e cura. Também foram abordados os estudos descritivos que tem por finalidade informar sobre a distribuição de um evento na população em termos quantitativos que podem ser identificados por prevalência ou incidência; no entanto, não há formação de grupo controle para comparar resultados.

Este estudo possibilitou também verificar que nas pesquisas em saúde qualquer que seja o delineamento utilizado, o mesmo deve estar de acordo com o tipo de problema a ser investigado, identificando-se desta maneira, o melhor tipo de design para a pesquisa a ser desenvolvida. É um desafio optar por este ou aquele método, mas identificá-lo é imprescindível para o desenvolvimento das pesquisas. O método é o caminho que deve ser traçado pelo pesquisador para alcançar os objetivos propostos na investigação e provar ou rejeitar as hipóteses.

Ressalta-se que, para investigar o tema ST, os delineamentos de pesquisa epidemiológica são pertinentes e devem ser analisados com profundidade para identificar o que mais se adéqua para o que se deseja investigar. Com certeza qualquer que seja o modelo adotado é uma possibilidade para descortinar o problema e propor soluções.

\section{Referências}

ALMEIDA FILHO, N. de; ROUQUAYROL, M. Z. Introdução à epidemiologia. 4. ed. Rio de Janeiro: Guanabara Koogan, 2006.
ALVES, R. B. Vigilância em saúde do trabalhador e promoção da saúde: aproximações possíveis e desafios. Cadernos de Saúde Pública, Rio de Janeiro, v. 19, n. 2, p. 319-322, 2003.

AUGUSTO, L.G. da S. Health and environmental surveillance: building the theme. Epidemiologia e Serviços de Saúde, Brasília, v. 12, n. 4, p.177-188, 2003.

BRASIL. Ministério da Saúde. Legislação em saúde: caderno de legislação em saúde do trabalhador. Brasília, DF: Ministério da Saúde, 2004.

. Ministério da Saúde. Coletânea de normas para o controle social no sistema único de saúde / MS. CNS. Brasília, DF: Ministério de Saúde, 2011. . Ministério da Saúde. Portaria no 3.120 de lo de julho de 1998. Aprova a instrução normativa de vigilância em saúde do trabalhador no SUS. Diário Oficial da União, Brasília, Seção 1, p.36, 2 jul.1998.

. Ministério da Saúde. Lei $n^{\circ} 8.080$, de 19 de setembro de 1990. Dispõe sobre as condições para a promoção, proteção e recuperação da saúde, a organização e o funcionamento dos serviços correspondentes e dá outras providências. Disponível em: <http://www.planalto.gov.br/ ccivil_03/Leis/L8080.htm>. Acesso em: 10 out. 2012.

BRIZ, T. Epidemiologia e saúde pública. Revista Portuguesa de Saúde Pública, Lisboa, p. 31-49, 2009. Número Especial.

CAMPBELL, D. T.; STANLEY, J. C. Experimental and quase experimental designs for research. Chicago: Rand McNally, 1966.

CERVO, A. L. et al. Metodologia científica. 6. ed. São Paulo: Pearson Prentice Hall, 2007.

DOCHERT, J. P. et al. Psychological treatment research in psychiatry. Washington: APA, 1993.

FLETCHER, R. H. et al. Epidemiologia clínica: elementos essenciais. Porto Alegre: Artes Médicas, 1996. 
GIL, A. C. Métodos e técnicas de pesquisa social. 6. ed. São Paulo: Atlas, 2008.

GIL, A. C. et al. Por que fazer pesquisa qualitativa em saúde? Caderno de Saúde, São Caetano do Sul, v. 1, n. 2, p. 5-19, jul./dez., 2006.

GREENLAND, S.; ROBINS, J. Accepting the limits of studies. American Journal of Epidemiology, v. 139, n. 8, p.769-771, 1994.

LIMA-COSTA, M. F.; BARRETO, S. M. Types of epidemiologic studies: basic concepts and uses in the area of aging. Epidemiologia e Serviços de Saúde, v. 12, n. 4, p. 189-201, 2003.

PELLIZZON, R. F.; POBLACION, D. A.; GOLDENBERG, S. Pesquisa na área da saúde: seleção das principais fontes para acesso à literatura científica, 2003. Acta Cirúrgica Brasileira, São Paulo, v. 18, n. 6, p. 493-496, 2003. Disponível em: $<$ http://www.scielo.br/pdf/acb/v18n6/a02v18n6.pdf >. Acesso em: 12 out. 2012.

PEREIRA, M. G.. Epidemiologia: teoria e prática. Rio de Janeiro: Guanabara Koogan, 2001.

U.S. DEPARTMENT OF HEALTH AND HUMAN SERVICES. Centers for Disease Control and Prevention (CDC). Principles of epidemiology in public health practice: an introduction to applied epidemiology and biostatistics. 3rd ed. Atlanta, 2012. Disponível em: <http://www.cdc.gov/osels/ scientific_edu/ss1978/SS1978.pdf>. Acesso em: 10 out. 2012.

ROUQUAYROL, M. Z.; GOLDBAUM, M. Epidemiologia, história natural e prevenção de doenças. In: ROUQUAYROL, M. Z.; ALMEIDA FILHO, N. Epidemiologia e saúde. 6. ed. Rio de Janeiro: Medsi, 2003. p. 17-35.

SILVA, L. A. A Vigilância permanente da saúde e o método epidemiológico. Revista de Saúde Pública de Santa Catarina, Florianópolis, v.1, n.1, p. 36-45, 2008.

SOARES, J. F.; SIQUEIRA, A.L. Introdução à estatística médica. Belo Horizonte: UFMG, 1999.
SOUZA, P. R. R. de. Epidemiologia e saúde do trabalhador. ENSP/Fiocruz, 2012. Disponível em:<http://www.imiarj.com.br/admin/pdf/ ARTIGO $\% 20$ EPIDEMIOLOGIA $\% 20$ E $\% 20$ S A \% C 3\% $83 \%$ C $5 \%$ A 1 D E \% 20 D O \% 20 TRABALHADOR.pdf >. Acesso em: 08 out. 2012. VASCONCELLOS, L. C. F.; RIBEIRO, F. S. N. Investigação epidemiológica e intervenção sanitária em saúde do trabalhador: o planejamento segundo bases operacionais. Cadernos de Saúde Pública, Rio de Janeiro, v. 13, n. 2, p. 269-275, 1997.

VASCONCELLOS, L. C. F. de; ALMEIDA, V. B.; GUEDES, D. T. Vigilância em saúde do trabalhador: passos para uma pedagogia. Trabalho Educação Saúde, 2009/2010. Rio de Janeiro, v. 7, n. 3, p. 445-462; Disponível em: <http://www.revista. epsjv.fiocruz.br/upload/revistas/r265.pdf >. Acesso em: 08 out. 2012.

Recebido em: 30 ago. 2013. Aceito em: 29 jun. 2014. 
